\title{
Nasoangiofibroma juvenil: manejo interdisciplinario de un caso
}

\author{
Juvenile nasoangiofibroma: an interdisciplinary case management
}

Vasti Evelyn Díaz Quiquia ${ }^{1^{*}}$

\begin{abstract}
RESUMEN
Introducción: El nasoangiofibroma juvenil (NAF) es un tumor nasofaríngeo benigno, altamente vascularizado, actualmente corresponde a una de cada 10.000 consultas otorrinolaringológicas y al $0,5 \%$ de todos los tumores de cabeza y cuello. Se caracteriza por presentar epistaxis recurrentes y obstrucción nasal. Caso clínico: Se presenta el caso de un adolescente de 16 años con epistaxis recurrentes y obstrucción nasal de un año de evolución, se le realizan TAC y RM donde se evidencia masa nasofaríngea derecha captadora de contraste, por lo que se programa embolización previa a la cirugía, se extrae tumor angiofibromatoso de $8 \times 4 \times 2 \mathrm{~cm}$, con posterior taponamiento anterior. El paciente se encuentra en su postoperatorio evolucionando favorablemente. Conclusión: El nasoangiofibroma juvenil es la neoplasia benigna más común de la nasofaringe con comportamiento maligno. El pronóstico es favorable en proporción a su reconocimiento y extirpación precoz. El diagnóstico precoz y el manejo multidisciplinario es la clave para el éxito.
\end{abstract}

Palabras clave: Nasoangiofibroma juvenil, Neoplasia vascular, Obstrucción nasal, Epistaxis.

\begin{abstract}
Introduction: The objective is to describe the case of juvenile nasopharyngeal angiofibroma (JNA) considered as a benign, highly vascularized nasopharyngeal tumor, currently corresponds to one of every 10,000 otorhinolaryngological consultations and $0.5 \%$ of all head and neck tumors. Clinical case: It is characterized by recurrent epistaxis and nasal obstruction. The case of a 16-year-old adolescent with recurrent epistaxis and a nasal obstruction of one year of evolution is presented. CT and MRI are performed where a contrast-enhanced nasopharyngeal mass is evident. Therefore, embolization is scheduled prior to surgery. Extracts angiofibromatoso tumor of $8 \times 4 \times 2 \mathrm{~cm}$, with previous tamponade. The patient is in his postoperative period evolving favorably. Conclusions: Juvenile nasoangiofibroma is the most common benign neoplasm of the nasopharynx with malignant behavior. The prognosis is favorable in proportion to its recognition and early extirpation. Early diagnosis and multidisciplinary management is the key to success.
\end{abstract}

Keywords: Juvenile nasoangiofibroma, Vascular neoplasia, Nasal obstruction, Epistaxis.

\footnotetext{
${ }^{1}$ Estudiante de Medicina Humana, Facultad de Ciencias de la Salud, Universidad Peruana Unión, Lima, Perú.
} 


\section{INTRODUCCIÓN}

El nasoangiofibroma juvenil (NAF) es un tumor nasofaríngeo benigno, altamente vascularizado, predominando su presentación en adolescentes masculinos (14- 18 años), con alta morbi-mortalidad por la hemorragia que genera y por la destrucción local secundaria a su patrón de crecimiento. (Hernández, 2011). Actualmente, corresponde a una de cada 10.000 consultas otorrinolaringológicas y al $0,5 \%$ de todos los tumores de cabeza y cuello (Mena, 2009).

La clínica se caracteriza por presentar epistaxis recurrentes, generalmente unilateral en estadíos tempranos, así como obstrucción nasal, respiración bucal, ronquidos nocturnos, rinorrea anterior y posterior, agravándose si el paciente presenta algún tipo de rinitis. (Mena, 2009). El diagnóstico es clínico y con ayuda de imágenes como TAC y RMN, y la biopsia está contraindicada en estos casos, la cual se reserva en casos de duda diagnóstica. La etiopatogenia sigue siendo desconocida, aunque la hipótesis más aceptada propone que el tumor sería resultado de un nido tumoral fibrovascular inactivo en la niñez, que se activa durante la pubertad por aumento del nivel de testosterona. (Contreras, 2012).

El manejo es multidisciplinario, principalmente quirúrgico, la embolización previa la cirugía ha disminuido la morbi-mortalidad intraquirúrgica al permitir mayor control vascular de la lesión (Burbano, 2011)

\section{CASO CLÍNICO}

Se presenta el caso de un adolescente de 16 años procedente de Huánuco, quien refiere inicio de cuadro hace 1 año caracterizado por epistaxis intermitentes, aproximadamente 3 veces por semana, espontáneos, de poca a moderada cantidad, que ceden a la compresión; seis meses después presenta obstrucción nasal en fosa derecha, la cual produce respiración bucal a predominio nocturno en inicio, pero llegando a intensificarse hasta presentarse durante el día, los síntomas se incrementan en intensidad y cada vez se hacen más frecuentes por lo que es referido al Hospital Nacional Guillermo Almenara Irigoyen por ser de mayor complejidad.

Al examen físico se evidencia presencia de tumoración color negro azulado que ocluye el 100\% de la luz localizada en área II Cottle, con secreción mucoide en fosa nasal derecha. El resto del examen físico fue negativo.

Se decide la hospitalización del paciente en el servicio de Otorrinolaringología, con el diagnóstico diferencial de Nasoangiofibroma, donde se le realizan exámenes auxiliares como Hemograma completo, AGA y otros, resultando estos en anemia moderada de 9,4 mg/dl, sin otra alteración aparente; así mismo se le realizan TAC y RMN (imagen 1,2 y 3).

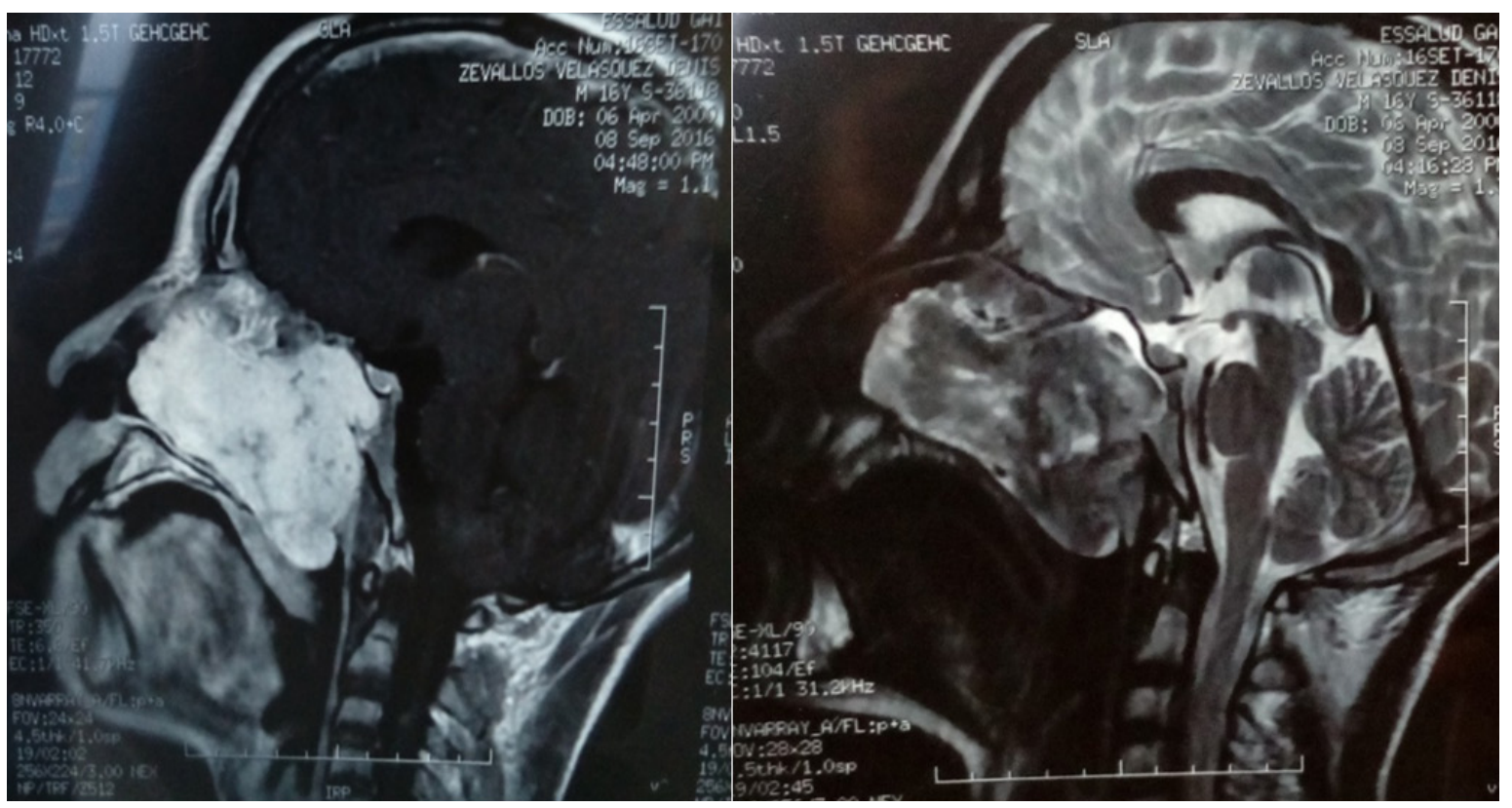

Imagen 1. Nasoangiofibroma que ocupa la fosa nasal e insinúa extensión a la fosa pterigomaxilar e infratemporal medial. 


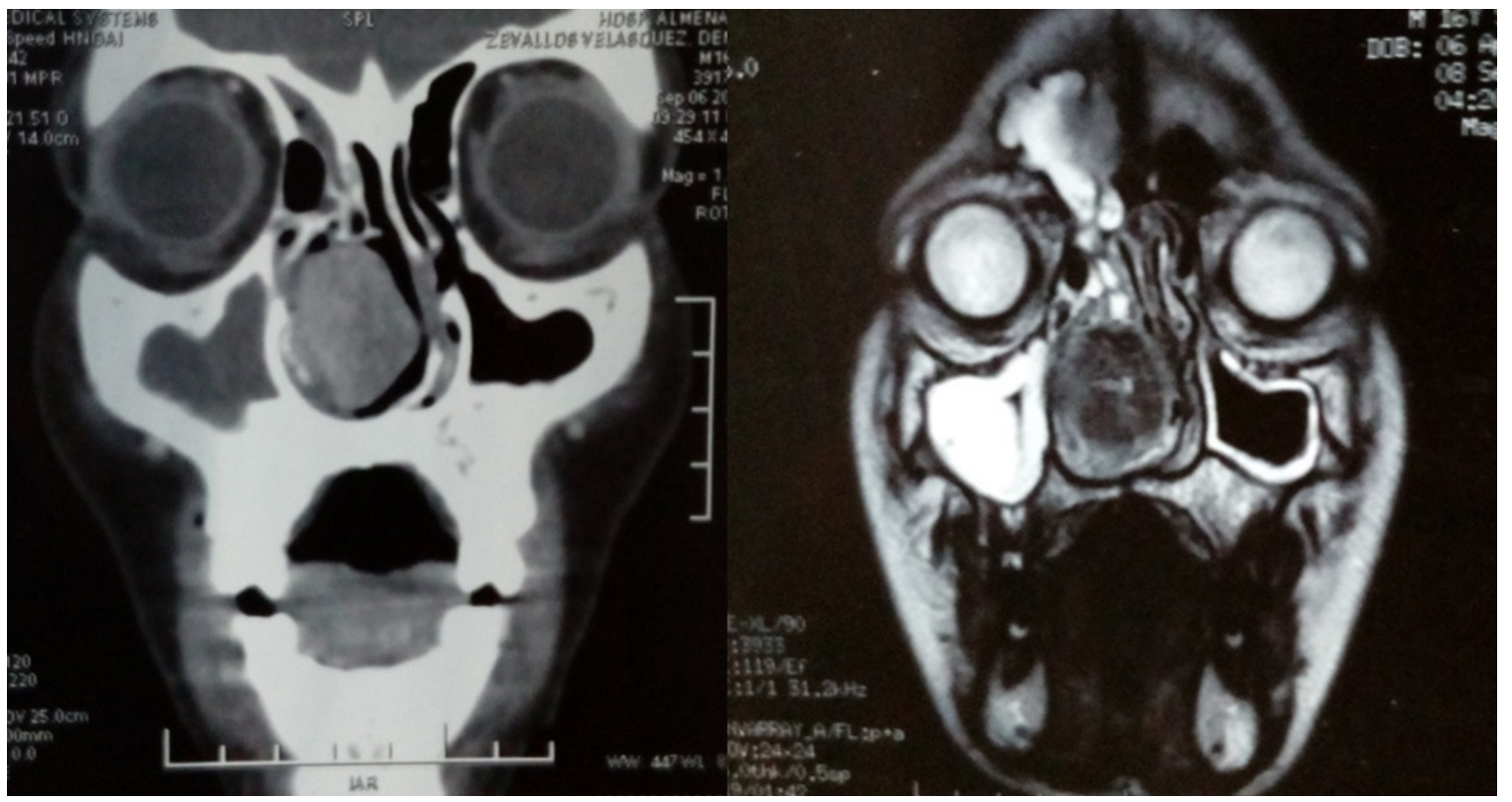

Imagen 2. Corte coronal donde se evidencia tumoración que invade seno maxilar derecho, desplazando tabique nasal. Asimismo se observa ocupación de seno frontal y maxilar derecho por posible sinusitis.

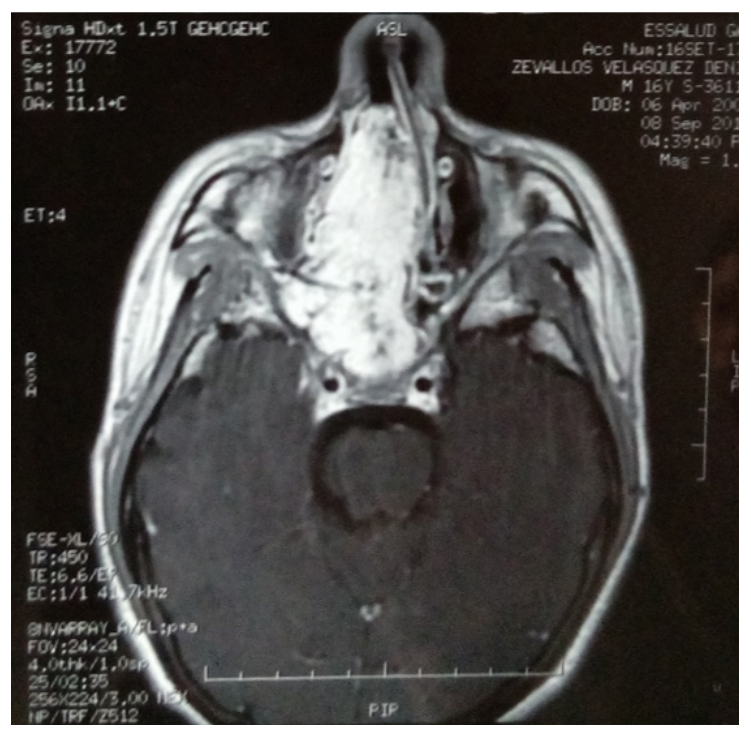

Imagen 3. Corte axial donde se evidencia masa captadora de contraste que desplaza tabique nasal izquierdo.

Se le realiza TAC y RM, donde se evidencia extensa lesión expansiva sólida, con realce de contraste que compromete el espacio naso y orofaríngeo, fosa nasal derecha, fosa pterigopalatina derecha, espacio prevertebral, senos esfenoidales, infiltra la base del esfenoides, seno cavernoso, conducto faringotimpánico y músculos pterigoideos derechos. Hallazgo que por sus características sugiere nasoangiofibroma juvenil, y pansinusitis aguda obstructiva, según descripción.

Se realiza la embolización por el departamento de Neuroradiología, la cual se efectúa bajo anestesia general; se ingresa por arteria femoral derecha, bajo técnica de Seldinger, y se realiza angiografía de arteria carótidas internas y externas bilaterales, donde se logra demostrar proceso expansivo retronasal derecho; se emboliza con partículas de Alcohol Polivilínico de 200 micras. El estudio de la arteria carótida externa derecha reveló la naturaleza hipervascular del tumor el que recibe aferencias de múltiples ramas esfenopalatinas.

La angiografía de control demostró la embolización total de la irrigación, con preservación de estructuras vasculares normales adyacentes. (Imagen 4) 


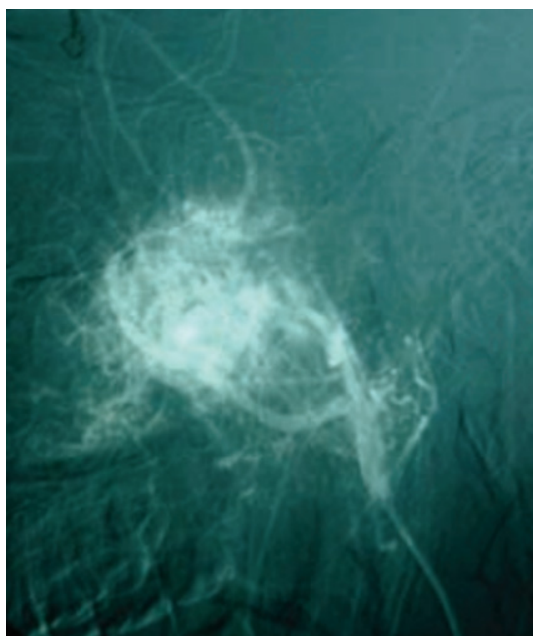

Imagen 4. Embolización de un Nasoangiofibroma. Una vez inyectado el medio de contraste se hace evidente la masa con vaso nutriente respectivo, Se procede a liberar las micropartículas para embolización del mismo.

El paciente ingresa a sala, bajo anestesia general, y se procede a realizar la cirugía tipo Degloving mas desarme facial, más resección de tumor nasofaríngeo; donde se halle tumor angiofibromatoso de $8 \times 6 \times 4 \mathrm{~cm}$ de consistencia firme multilobulado que se extiende lateralmente a fosa pterigomaxilar derecha, fosa infratemporal, medial y superiormente se extiende a seno esfenoidal derecho con múltiples adherencias firmes a estructuras descritas, sangrado total de $800 \mathrm{cc}$, se visualiza y preserva nervio infraobitario derecho. Se procede a realizar osteosíntesis con miniplacas y tornillos sistema 1.5 , fijación de drenes en hilos de reposo para tapón posterior. Se envía la pieza quirúrgica a Patología.

La duración de la cirugía fue de 5 horas con 50 minutos, el paciente sale de sala entubado, con insuficiencia respiratoria aguda post quirúrgica, anemia aguda y lactato con tendencia a incrementarse (2.0 - 2.3 - 4.5), por lo que es transferido al servicio de UCI (Unidad de cuidados intensivos), donde se le realiza transfusión de paquete globular, ascendiendo la hemoglobina a $12.6 \mathrm{mg} / \mathrm{dl}$. El último examen de gases arteriales demostró estabilización del medio interno con un nivel de lactato de $0.7 \mathrm{mmol} / \mathrm{L}$.

Dos días después regresa al servicio de Otorrinolaringología, ya estable donde se le administra cobertura antibiótica con ceftriaxona $2 \mathrm{gr}$ cada 24 horas EV, clindamicina $600 \mathrm{mg}$ cada 8 horas EV; así mismo Dexametasona $4 \mathrm{mg}$ cada 24 horas EV. Siete días después de la cirugía se extrae tapón nasal, evidenciándose fosas nasales permeables, no sangrantes. El paciente muestra mejoría; actualmente se encuentra en su postoperatorio evolucionando favorablemente.

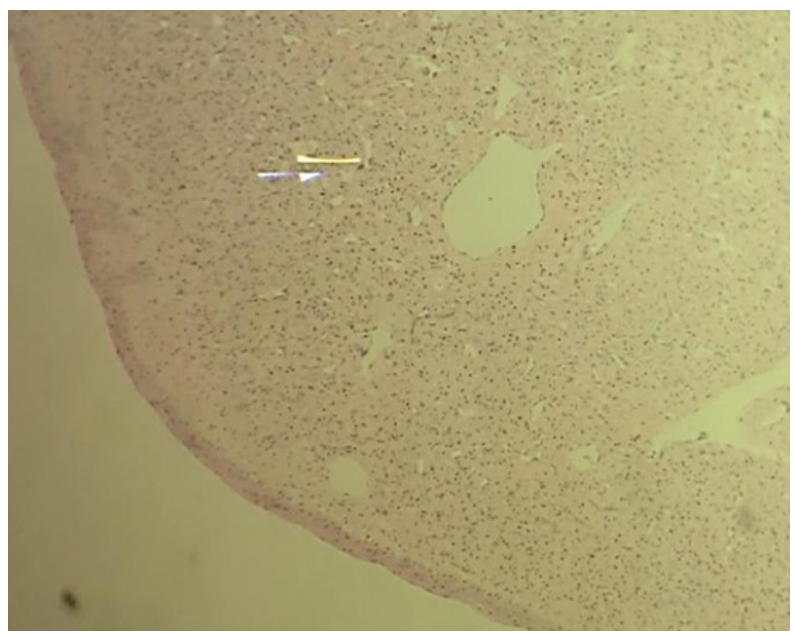

Imagen 5. Angiofibroma nasofaríngeo (H-E x 10X) Se observa estroma fibroso con múltiples vasos sanguíneos. 


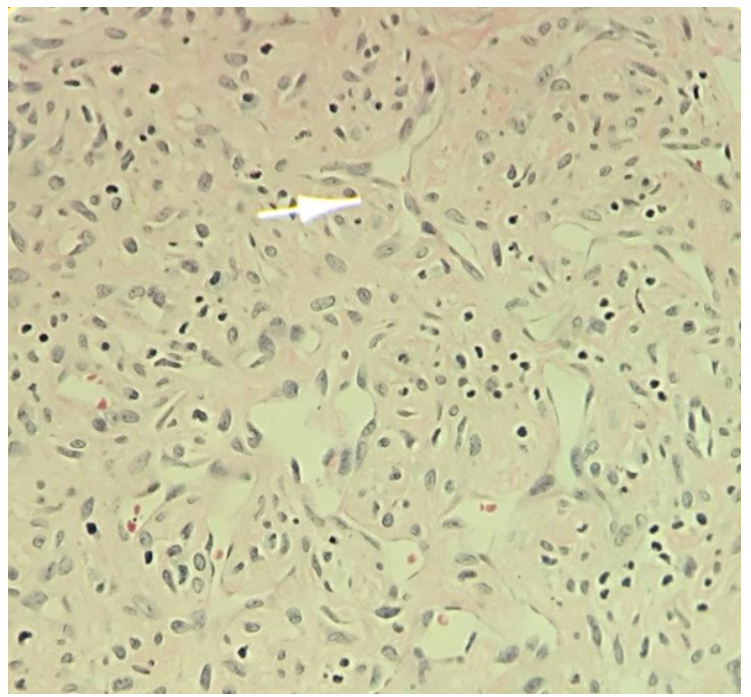

Imagen 6. Angiofibroma nasofaríngeo (H-E x 40X) Se observan vasos sanguíneos desorganizados de tamaña variable, con endotelio reactivo, estroma laxo con fibroblastos, células fusiformes y linfocitos.

\section{DISCUSIÓN}

El nasoangiofibroma juvenil es la neoplasia benigna más común de la nasofaringe con comportamiento maligno. (Ley-Mandujano, 2014). Una de las manifestaciones clínicas es la epistaxis que pone en riesgo la vida por su magnitud y frecuencia. En nuestro caso, el paciente mostró anemia moderada de 9,4 mg/dl posterior a los sangrados nasales, llegando a estabilizarse a 12,6 mg/dl después de la transfusión. El angiofibroma es una patología de difícil diagnóstico en los primeros estadios, es un reto diagnóstico y terapéutico, generalmente se lo diagnostica en los últimos estadios III y IV (clasificación de Chandler)7, como en el caso que presentó estadio III de Chandler. (Tabla 1) El paciente presentó la mayoría de las manifestaciones clínicas característicos de esta patología, como la epistaxis y la obstrucción nasal.

\begin{tabular}{|l|l|}
\hline \multicolumn{2}{|l|}{$\begin{array}{l}\text { Tabla 1. Clasificación de angiofibroma nasofaríngeo } \\
\text { de Chandler }\end{array}$} \\
\hline I & Tumor localizado en la nasofarínge \\
\hline II & $\begin{array}{l}\text { Tumor se extiende a fosa nasal y/o seno } \\
\text { esfenoidal }\end{array}$ \\
\hline III & $\begin{array}{l}\text { Tumor que se extiende a uno o más de las } \\
\text { siguientes estructuras: seno maxilar, etmoidal, } \\
\text { fosa pterigomaxilar, fosa infratemporal, órbita } \\
\text { y/o mejilla }\end{array}$ \\
\hline IV & Tumor que invade la cavidad craneal \\
\hline
\end{tabular}

Existen recidivas de un 5 a $37 \%$ en especial los tumores que invaden la estructura ósea de la base del cráneo, por eso es importante aplicar una exéresis meticulosa e intensificar el control permanente. 7
Dentro de los diagnósticos diferenciales se pueden plantear tumoraciones benignas, como posibilidades etiológicas y a descartar mediante estudio anatomopatológico, el fibroma, adenoma o el angiofibroma. No obstante, en estos casos no debemos dejar de tener en cuenta en segundo lugar, la tumoración maligna del tipo linfosarcoma, que también es frecuente en los jóvenes, y cuya evolución no tendría el mismo pronóstico. (Tapia, 2013).

A lo largo del tiempo, se han implementado varias opciones terapéuticas incluyendo la cirugía, la radiación, la radioterapia, quimioterapia, crioterapia y terapia Hormonal9. Sin embargo, la modalidad terapéutica que ofrece los mejores resultados es el manejo quirúrgico, aunque se hayan descrito alguna tasa de éxito con la radioterapia y los antineoplásicos.

El proceder quirúrgico constituye el principal punto de la terapéutica en el angiofibroma juvenil nasofaríngeo, y para esto se recomienda contar con la transfusión de sangre necesaria por la tendencia a la hemorragia. En el angiofibroma juvenil nasofaríngeo hemorrágico, la epistaxis puede ser el primer síntoma o signo, aunque ante todo paciente con sintomatología obstructiva nasal, debe realizarse un riguroso examen rinoscópico posterior. La radiología de nariz y senos para-nasales, son exámenes primordiales en el diagnóstico y tratamiento del angiofibroma juvenil nasofaríngeo. (Gualberto, 2013).

Un abordaje ideal debe considerar la extensión del tumor, la suplencia vascular y permitir lograr buen control vascular. (Tosun, 2006). 
Cabe resaltar que nuestro paciente fue ingresado en el servicio de Otorrinolaringología, sin embargo era indispensable la participación de Neuroradiología para la embolización pre-quirúrgica, la cual pudo delimitar las zonas anatómicas que invadía el tumor, facilitando así el manejo quirúrgico.

La embolización y las nuevas técnicas quirúrgicas disminuyen la morbilidad y la recurrencia, con resultados de cura cercanas al $100 \%$ en casos de resección extracraneal,

$70 \%$ en tumores con extensión intracraneal y del $90 \%$ en segundas cirugías por recurrencia. (Browne, 2000). La presencia de los médicos intensivistas en el manejo post-quirúrgico fue primordial para la estabilización y recuperación del paciente.

En conclusión el pronóstico es favorable en proporción a su reconocimiento y extirpación precoz.12 El diagnóstico precoz y el manejo multidisciplinario es la clave para el éxito en estos casos.

\section{Agradecimientos}

Se agradece el asesoramiento de este trabajo al Dr. Luis Guillermo Peralta Valderrama, Especialista en Otorrinolaringología, y profesor principal de la Escuela de Medicina Humana de la Universidad Peruana Unión. Así mismo al Dr. Omar Gonzáles, jefe del Servicio de Otorrinolaringología del Hospital Nacional Guillermo Almenara Irigoyen. Un agradecimiento especial a la Dra. Jessica Mathews, Patóloga del Hospital Nacional Guillermo Almenara Irigoyen.

\section{Correspondencia}

Vasti Diaz Quiquia

EP Medicina Humana, Universidad Peruana Unión. Carretera Central Km 19.5 Ñaña. Lima Perú e-mail: revistasalud@upeu.edu.pe

\section{REFERENCIAS BIBLIOGRÁFICAS}

Browne, S. (2000). Temporal Approach for Resection of Juvenile Nasopharyngeal Angiofibromas. Laryngoscope; 110:1287-1293.

Burbano, K. Barros, C. González. (2011). Reporte de caso clínico: fibroangioma nasal juvenil. Rev. Med. FCMUCSG, Vol.17 N². Págs. 126-131 ISSN - 1390-0218.

Contreras et al (2012). Nasoangiofibroma. Evidencia Médica e investigación en salud. Vol. 5, Núm. 3 • Julio-Setiembre 2012 pag 95-98.

Gualberto. J. (2012). Angiofibroma juvenil nasofaríngeo. Revista Cubana de Medicina General Integral. 2012;28(2):87-92

Hernández, S. Hernández (2011). Nasoangiofibroma juvenil: una revisión actualizada del diagnóstico, clasificación y tratamiento. Acta de Otorrinolaringología \& Cirugía de Cabeza y Cuello. Volumen 39 Número 3.

Ley-Mandujano S (2014). Campos-Navarro LA. Patrón histológico del nasoangiofibroma en pacientes del Centro Médico Nacional La Raza. An Orl Mex;59:165-170.

Mena, F. Mena, H. Knecht, J. Roig. (2009). Nasoangiofibroma juvenil: Revisión de casos de pacientes en edad pediátrica. Pediatría. (Asunción), Vol. 36; № 2.

Mena, G. Bogado, C. Klassen.(2009). Nasoangiofibroma juvenil: Nuestra experiencia en los últimos 10 años y revisión de la literatura. Rev. Otorrinolaringol. Cir. Cabeza Cuello v.69 n.3 Santiago.

Pryor, E. Moore, J. Kasperbauer. Endoscopic versus Traditional Approaches for Excision of Juvenile Nasopharyngeal Angiofibroma. Laryngoscope 2005; 115 (7): $1201-7$

Tapia L. (2013). Cile. Angiofibroma Juvenil. SCIENTIFICA v.11 n.1 La Paz.

Tosun, C. Ozer, M. Gerek, S. Yetiser. (2006). Surgical Approaches for Nasopharyngeal Angiofibroma: Comparative Analysis and Current Trends. The journal of craniofacial surgery; 17(1): 15-20.

Recibido: 06/11/2016

Aceptado: 04/04/2017 


\section{NORMAS GENERALES PARA PRESENTACIÓN DE ARTÍCULOS}

La Revista Científica de Ciencias de la Salud, publica artículos originales empíricos y teóricos, comunicaciones originales cortas, reportes de casos en las áreas de Enfermería, Psicología, Nutrición y Medicina. Todo investigador que desee colaborar en la revista deberá considerar las siguientes indicaciones:

1. Los manuscritos deben enviarse a la revista en línea ingresando: http://revistascientificas.upeu.edu.pe/ index.php/rc_salud, o escribir al correo electrónico: revistasalud@upeu.edu.pe. Deberán ser originales e inéditos y se asume que toda persona que figure como autor ha dado su conformidad para que el manuscrito sea publicado.

2. Los manuscritos recibidos para publicación son sometidos a un proceso de revisión por pares. El manuscrito es enviado a dos revisores nacionales o extranjeros, pares de los investigadores, quienes realizan las correcciones $\mathrm{u}$ observaciones que sean pertinentes; luego el manuscrito es devuelto a los autores para que realicen las correcciones o respondan a las observaciones realizadas. En el caso que los revisores emitan opiniones contradictorias, se envía a un tercer revisor. Luego, el manuscrito es sometido a una revisión de la redacción y finalmente pasa a la diagramación. La versión diagramada tal como será publicada se envía a los autores para su corrección o aprobación.

3. El manuscrito debe ser presentado mediante una carta dirigida al Editor Jefe de la Revista Científica de Ciencias de la Salud, firmada por uno de los autores, solicitando la evaluación para ser considerada su publicación. La carta debe incluir el título del trabajo, el nombre completo de los autores y tipo de trabajo. Junto con la carta de presentación se debe entregar un original impreso, y la versión electrónica se debe enviar a la página web http://revistasinvestigacion.upeu.edu.pe/ index.php?journal=rc_salud

4. Se debe adjuntar una declaración jurada firmada por todos los autores (DECLARACION JURADA Autores Artículos), que el artículo presentado es propiedad intelectual de los autores, que no ha sido publicado, ni presentado para evaluación en otra revista, cediendo los derechos de autor a la Revista Científica de Ciencias de la Salud una vez que el artículo sea publicado.

5. El artículo debe ser redactado en castellano, portugués o inglés, impreso en papel bond blanco de medida ISOA4 (212x297 mm), en una sola cara, tipo de letra Times New Roman, tamaño de fuente 12 picas, a doble espacio, con márgenes de $25 \mathrm{~mm}$.
Cada parte del artículo debe empezar en página aparte, numeradas en forma consecutiva. En el caso de citas textuales, el párrafo correspondiente dentro del texto se destacará colocándolo entre comillas o escribiéndolo con letra itálica. Las tablas y figuras con su título correspondiente, se colocan al final del texto en páginas aparte; no deben ser insertados en el texto.

\section{ESQUEMAS DE PRESENTACIÓN:}

Investigaciones originales: Resumen; Abstract; Introducción; Material y métodos; Resultados; Discusión; Referencias bibliográficas; Tablas y figuras. La extensión total del manuscrito, incluyendo las referencias bibliográficas, no debe ser mayor de 14 páginas escritas en una sola cara sin incluir tablas, gráficos y figuras. Se acepta como máximo de diez tablas y figuras; el número máximo de referencias bibliográficas es 40 .

\section{Comunicaciones cortas:}

Contienen las mismas partes que una investigación original. La extensión total del manuscrito, incluyendo las referencias bibliográficas, no debe ser mayor de 6 páginas escritas en una sola cara, sin incluir tablas y figuras. Se acepta como máximo de seis tablas y figuras; el número máximo de referencias bibliográficas es 20 .

\section{Reporte de casos:}

Resumen, Abstract; Introducción, Presentación del caso, Conclusiones, Referencias bibliográficas. La extensión total del trabajo, incluyendo las referencias bibliográficas, no debe ser mayor de seis páginas escritas en una sola cara, sin incluir tablas, gráficos y figuras. Se acepta como máximo de seis tablas, gráficos y figuras; el número máximo de referencias bibliográficas es 20 .

7. Los resúmenes (en el idioma original e inglés) se presentan cada una en hoja aparte, teniendo una extensión máxima de 250 palabras y deben ser escritos en un solo párrafo. Al final se deben agregar 3 a 5 palabras clave o key words, que ayuden a clasificar el artículo.

8. La información a los autores e instrucciones para la presentación de manuscritos, donde se detallan las normas de formato y contenido de los artículos que se presentarán a la Revista, se pueden obtener de la página electrónica: http://revistasinvestigacion. upeu.edu.pe/index.php?journal=rc_salud

9. El estilo de la revista se basa en la $6^{\circ}$ edición revisada del Manual de Publicaciones de la APA. 
10. Una vez que el autor ha sido notificado acerca de la aprobación de su trabajo para su publicación, no se administrarán modificaciones ni agregados.

11. Los nombres y direcciones de correo-e introducidos en esta revista se usarán exclusivamente para los fines declarados por esta revista y no estarán disponibles para ningún otro propósito u otra persona.
12. Política de acceso abierto: Esta revista provee acceso libre inmediato a su contenido bajo el principio de que hacer disponible gratuitamente la investigación al público, siempre y cuando cite correctamente dentro de su manuscrito, el articulo consultado, lo cual fomenta un mayor intercambio de conocimiento global. 